\title{
Clinical and Radiographic Outcomes of Femoral Head Fractures Associated with Traumatic Hip Dislocations
}

\author{
Michael A Del Core ${ }^{1}$, Bruno Gross ${ }^{2}$, Junho Ahn ${ }^{3}$, Stephen Blake Wallace ${ }^{4}$, Adam Starr ${ }^{5}$
}

\begin{abstract}
Background: Femoral head fractures are an uncommon but severe injury. These high-energy injuries typically occur in association with traumatic hip dislocations. Initial treatment includes urgent concentric reduction; however, controversy exists regarding specific fracture management. The well-known complications of avascular necrosis (AVN), posttraumatic arthritis (PTA), and heterotrophic ossification can leave patients with a significant functional loss of their affected hip. The purpose of this study is to evaluate the clinical and radiographic outcomes of femoral head fractures.

Methods: A retrospective review was performed at our institution assessing all patients who presented from 2007 to 2015 with a femoral head fracture associated with a hip dislocation and at least 6 months of clinical and radiographic follow-up. Twenty-two patients met our inclusion criteria. There were 15 males and 7 females with an average age of 36 years (range: 17-55). The average follow-up time was 18 months (range: 6-102). Fractures were classified according to the Pipkin classification. The Thompson and Epstein score was used to determine functional outcomes.

Results: There were five, Pipkin I, 3 Pipkin II, 0 Pipkin III, and 14 Pipkin IV, femoral head fractures. Sixteen patients were successfully closed reduced in the emergency department (ED) and six patients required open reduction after failed reduction in the ED. Four patients (18\%) were successfully treated with closed reduction alone and 18 patients (82\%) required operative intervention. Of those undergoing operative intervention, one patient underwent excision of the femoral head fragment, seven underwent open reduction internal fixation (ORIF) of the femoral head, nine underwent ORIF of the acetabulum, and one underwent ORIF of the femoral head and the acetabulum. Nine patients (41\%) had an uneventful postoperative course. Two patients (9\%) developed AVN, both requiring total hip arthroplasty (THA). Five patients (23\%) developed PTA, two eventually requiring a THA. Two patients $(9 \%)$ had sciatic nerve palsy. One patient (5\%) developed a postoperative infection and four patients (18\%) developed heterotrophic ossification (HO), none requiring operative treatment. Two patients (9\%) had persistent anterolateral (AL) thigh numbness. Overall functional results were excellent in six patients (27\%), good in six (27\%), fair in seven (32\%), and poor in three patients (14\%). Four patients (18\%) required a THA.

Conclusion: Femoral head fractures are a rare injury with well-known complications. Early diagnosis and concentric reduction are the prerequisites for successful treatment. This study adds to the growing literature on femoral head fractures associated with hip dislocations in efforts to define treatment plans and to guide patient expectations.

Keywords: Avascular necrosis, Complication, Femoral head fracture, Heterotrophic ossification, Hip dislocation, Outcome, Pipkin, Posttraumatic arthritis.

Strategies in Trauma and Limb Reconstruction (2019): 10.5005/jp-journals-10080-1416
\end{abstract}

\section{INTRODUCTION}

Femoral head fractures are a rare but severe injury. Hip dislocations accompany a majority of these fractures with an incidence ranging from $5 \%$ to $15 \% .^{1-8}$ These fracture dislocations are seen in highenergy trauma, most commonly motor vehicle accidents, when a flexed knee strikes the dashboard and transmits an axial force onto a flexed hip. The Pipkin classification is the most commonly used system to evaluate these fractures. ${ }^{9}$ Pipkin subdivided these injuries based on the position of the femoral head fracture line relative to the fovea and associated femoral neck and acetabulum fractures (Table 1).

The initial workup of these injuries at our institution is as follows. Femoral head fracture-dislocations are identified on a standard pelvic radiograph taken during the initial trauma work up. Once identified, closed reduction under sedation is attempted in the ED. If closed reduction is not obtained, open reduction is performed as urgently as possible. Postreduction radiographs and computerized tomography are obtained to assess for intra-articular fragments, associated fractures, and to confirm anatomic reduction. Further surgical treatment is required for those fracture dislocations that are irreducible, unstable, or with large intra-articular loose fragments.

\begin{abstract}
${ }^{1-5}$ Department of Orthopaedic Surgery, University of Texas Southwestern, Dallas, Texas, USA

Corresponding Author:Michael ADelCore,Department ofOrthopaedic Surgery, University of Texas Southwestern, Dallas, Texas, USA, Phone: +1-402-250-5595, e-mail: madelcore@gmail.com

How to cite this article: Del Core MA, Gross B, Ahn J, et al. Clinical and Radiographic Outcomes of Femoral Head Fractures Associated with Traumatic Hip Dislocations. Strategies Trauma Limb Reconstr 2019;14(1):6-10.
\end{abstract}

Source of support: Nil

Conflict of interest: None

Patients with successfully reduced fractures without the above CT findings are made toe-touch weight bearing for 6 weeks with early range of motion encouraged but limited to $70^{\circ}$ flexion, neutral adduction, and neutral internal rotation. Serial radiographs at each clinic visit are viewed and progressive weight bearing was started once clinical and radiographic signs dictate sufficient healing.

Femoral head fractures are associated with serious wellknown complications of AVN, PTA, sciatic nerve palsy, and HO. These complications can lead to unfavorable and potentially 
Table 1: Pipkin classification

\begin{tabular}{ll}
\hline Type & Description \\
\hline I & $\begin{array}{l}\text { Dislocation with fracture of the femoral head caudad to the } \\
\text { fovea capitis femoris }\end{array}$ \\
II & $\begin{array}{l}\text { Dislocation with fracture of the femoral head cephalad to } \\
\text { the fovea capitis femoris }\end{array}$ \\
III $\quad$ Type I or II injury associated with fracture of the femoral neck \\
IV $\quad$ Type I or II injury associated with fracture of the acetabulum
\end{tabular}

disabling patient outcomes. The limited incidence of this injury has led to a lack of large high-quality studies and a deficiency in definitive treatment protocols. The purpose of the present study is to report the functional outcomes and complications of all treatment modalities for femoral head fractures associated with hip dislocations.

\section{Methods}

We retrospectively reviewed the records of all patients who sustained a hip dislocation associated with a femoral head fracture treated at our level 1 trauma center from 2007 to 2015. Inclusion criterion included: (1) hip dislocation and associated femoral head fracture; (2) age 16-65; and (3) follow up $\geq 6$ months. Patients were excluded if they presented with a pathological fracture or had any previous injury to the affected hip. Patients were classified according to the Pipkin classification system. ${ }^{9}$

A total of 22 patients met our inclusion criteria. There were 15 men (68\%) and 7 women (32\%) with an average age at the time of injury of 36 years (range: 17-55). Fourteen patients sustained their fracture dislocation from a motor-vehicle accident, four from a motorcycle accident, two from a fall, one from an all-terrain vehicle (ATV), and one from a motor-pedestrian accident. All patients presented with posterior dislocations. There were 5 (23\%) Pipkin I fractures, 3 (14\%) Pipkin II, 0 (0\%) Pipkin III, and 14 (64\%) Pipkin IV fractures. Patient demographics and classification are listed in Table 2.

Definitive treatment and timing varied between patients dependent upon the fracture pattern and associated injuries. The following general guidelines were used to determine the need for operative intervention: nonreducible fracture dislocations, nonconcentric reduction, hip instability after reduction, or large

Table 2: Demographics and classification

\begin{tabular}{lc}
\hline & Number of patients (\%) \\
\hline Age (mean) & 36 years \\
Sex & \\
Males & $15(68)$ \\
Females & $7(32)$ \\
Mechanism of injury & \\
Motor vehicle accident & $14(64)$ \\
Motorcycle accident & $4(18)$ \\
Fall & $2(9)$ \\
Motor-pedestrian accident & $1(4.5)$ \\
All-terrain vehicle accident & $1(4.5)$ \\
Classification & \\
Pipkin I & $5(23)$ \\
Pipkin II & $3(14)$ \\
Pipkin III & $0(0)$ \\
Pipkin IV & $14(64)$ \\
\hline
\end{tabular}

intra-articular fragments. Patient outcomes and complications were determined based on a review of the clinic and radiographic results from their last clinical visit. The Brooker classification was used to evaluate $\mathrm{HO}$ formation. ${ }^{10}$ Functional outcomes were graded according to the Thompson and Epstein score. ${ }^{11}$ The average follow-up period was 18 months (range: 6-102).

\section{Results}

All patients presented with posterior hip dislocations and underwent an attempted closed reduction in the ED. This reduction was successful in 16 patients (73\%) and 6 patients (27\%) required an open reduction after failed closed reduction. The average time from injury to reduction was 321 minutes (range: 9-760). Traction was used in 15 patients (68\%) to offload the joint until surgery could be performed.

Four patients (18\%) were successfully treated with closed reduction alone. One patient (5\%) underwent excision of the femoral head fragment. Seven patients (32\%) underwent open reduction and internal fixation of the femoral head. Nine patients (41\%) underwent open reduction and internal fixation of the acetabulum. Two of these nine patients also underwent excision of the femoral head fragment. One patient $(5 \%)$ underwent open reduction and internal fixation of the femoral head and the acetabulum. The average time from injury until surgery was 58 hours (range: $8-168)$. An anterior approach was used in 5 patients (28\%) and a posterior approach was used in 13 patients (72\%). Postoperatively 17 patients (77\%) were made foot-flat weight-bearing for 12 weeks with hip precautions and an abduction pillow and 5 patients (23\%) were made nonweight-bearing with an abduction brace. This was determined at the discretion of the surgeon. Seven patients (32\%) underwent postoperative radiotherapy for $\mathrm{HO}$ prophylaxis.

Nine patients (41\%) had an uneventful postreduction course. Two patients developed AVN for an overall incidence of $9 \%$. Both of these patients required eventual conversion to a THA. Five patients (23\%) had radiographic criteria of PTA at their latest clinical follow-up. Two of these five patients required conversion to a THA. The incidence of sciatic nerve palsy was $9 \%$ (2/22 patients). The peroneal distribution was affected in both of these individuals. Complete resolution was seen in one patient and the other had continued palsy at their latest clinical follow-up. Postoperative infection occurred in one patient $(5 \%)$ requiring irrigation and debridement and a course of antibiotics. Four patients (18\%) developed HO. None of these patients required operative intervention. This was graded as Brooker I in all four patients. Two patients (9\%) had persistent AL thigh numbness at their last clinic visit. Both patients had undergone an anterior approach.

Clinical and radiographic data were reviewed for all patients at their latest clinical follow-up and graded according to the Thompson and Epstein classification. ${ }^{11}$ The overall results were excellent in six patients (27\%), good in six (27\%), fair in seven (32\%), and poor in three patients (14\%). One of the patients with a fair result and all three of the patients with poor results underwent eventual conversion to a THA. Overall treatments, complications, and outcomes are given in Table 3. Results based on the Pipkin classification are given in Table 4.

\section{Discussion}

Femoral head fractures associated with hip dislocations are rare but consequential injuries. ${ }^{21-31}$ It is agreed that early diagnosis and prompt concentric reduction are the initial management 
Table 3: Overall treatments, complications, and outcomes

\begin{tabular}{ll}
\hline & Number of patients (\%) \\
\hline Treatment & $4(18)$ \\
Closed reduction alone & $1(5)$ \\
Excision of fragment & $7(32)$ \\
ORIF femoral head & $9(41)$ \\
ORIF acetabulum & $1(5)$ \\
ORIF femoral head and acetabulum & \\
Complications & $9(41)$ \\
None & $2(9)$ \\
AVN & $5(23)$ \\
PTA & $2(9)$ \\
Sciatic nerve injury & $1(5)$ \\
Infection & $4(18)$ \\
HO & $2(9)$ \\
AL thigh numbness & \\
Thompson and Epstein score & $6(27)$ \\
Excellent & $6(27)$ \\
Good & $7(32)$ \\
Fair & $3(14)$ \\
Poor & \\
THA & $4(18)$ \\
Yes & $18(82)$ \\
No & \\
\hline
\end{tabular}

goals. Regarding definitive treatment, there is no clear consensus in part due to the lack of high-quality randomized controlled trials with validating outcome measures. Most studies present relatively small patient populations with short-term follow-up. Results and outcomes of these injuries have varied and are limited to a few quality studies. Giannoudis et al. in a 2009 systematic review found 40 excellent (14.3\%), 111 good (39.8\%), 54 fair (19.3\%), and 74 poor (26.5\%) overall results regardless of fracture type according to the Thompson and Epstein criteria. ${ }^{8}$ Our study found an overall excellent rate of $27 \%$, a good rate of $27 \%$, a fair rate of $32 \%$, and a poor rate of $14 \%$. Specific treatments and outcomes can be further explored by grouping according to the Pipkin classification.

Regarding Pipkin type I fractures, treatment varies among closed treatment, surgical excision, or fragment fixation. Treatment decisions are made based on fragment size, location, and comminution. Giannoudis et al. in their systematic review found that fragment excision trended toward better outcomes compared to open reduction and internal fixation $(p=0.07){ }^{8}$ Chen et al. found statistically better outcomes ( $p=0.032$ ) with fragment excision then closed reduction in a randomized prospective study looking at Pipkin I femoral head fractures. ${ }^{12}$ Park et al. explored Pipkin I fractures in regard to fragment size and location. With large fracture fragments, they found excellent or good results with excision of fragment in 7 patients (50\%) and excellent or good results with fixation in 19 patients (82.6\%). ${ }^{13}$ We elected to perform fixation in three out of five of our Pipkin I fractures given the fragment size. Our results were poor, fair, and good in these three patients. Regarding the other two patients, one underwent fragment excision and another was treated conservatively. The conservative treatment was our only Pipkin I fracture with an excellent outcome.

With Pipkin II fractures, there is a trend toward operative fixation as opposed to fragment excision as there is a larger portion of the weight-bearing surface of the femoral head involved. ${ }^{8}$ Holmes et al. performed a cadaveric biomechanical study to measure the influence of head excision on load transmission in the hip. For Pipkin type II fractures, the authors found an increased mean pressure across the center of the acetabulum, postulating that this could be responsible for the worse outcomes seen with fragment excision. ${ }^{14}$ Chen et al. performed a randomized prospective study of 24 patients undergoing either closed reduction or ORIF of Pipkin II femoral head fractures. The authors found functional outcomes to be worse for the conservative group compared to the surgical group $(p=0.37) .^{15}$ All three of our Pipkin II fractures underwent open reduction and internal fixation of the fracture fragment with results ranging from fair to excellent.

Table 4: Treatments, complications, and outcomes based on the Pipkin classification

\begin{tabular}{|c|c|c|c|c|}
\hline Fracture type & Treatment & Complications & Outcome* & THA \\
\hline \multirow[t]{4}{*}{ Pipkin I $(n=5)$} & Closed treatment alone: 1 & None: 2 & Poor: 1 & No: 4 \\
\hline & Excision of fragment: 1 & AVN: 1 & Fair: 2 & Yes: 1 \\
\hline & ORIF femoral head: 3 & PTA: 1 & Good: 1 & \\
\hline & & $\mathrm{HO}: 2$ & Excellent: 1 & \\
\hline \multirow[t]{4}{*}{ Pipkin II $(n=3)$} & ORIF femoral head: 3 & None: 1 & Poor: 0 & No: 3 \\
\hline & & PTA: 1 & Fair: 1 & Yes: 0 \\
\hline & & AL thigh numbness: 1 & Good: 1 & \\
\hline & & & Excellent: 1 & \\
\hline \multirow[t]{7}{*}{ Pipkin IV $(n=14)$} & Closed treatment alone: 3 & None: 6 & Poor: 2 & No: 11 \\
\hline & ORIF femoral head: 1 & AVN: 1 & Fair: 4 & Yes: 3 \\
\hline & ORIF acetabulum: $9^{* *}$ & PTA: 3 & Good: 4 & \\
\hline & $\begin{array}{l}\text { ORIF femoral head and ac- } \\
\text { etabulum: } 1\end{array}$ & HO: 2 & Excellent: 4 & \\
\hline & & Sciatic nerve injury: 2 & & \\
\hline & & Infection: 1 & & \\
\hline & & AL thigh numbness: 1 & & \\
\hline
\end{tabular}

$n$ is the number of patients

*Based on the Thompson and Epstein grading scale

**Two of these patients also underwent excision of femoral head fragment 
Pipkin III is the least frequent fracture type with treatment options ranging from open reduction and rigid fixation to arthroplasty depending on patient age and other variables. We did not encounter any Pipkin III in our population. Pipkin IV fractures are unique in that they involve both the femoral head and the acetabulum. This injury is particularly challenging, as the operating surgeon must decide whether to address the acetabulum, femoral head, or both. Machetti et al. showed that Pipkin III and IV injuries had statistically significant worse outcomes than those who sustained Pipkin I and II injuries $(p<0.02) .{ }^{16}$ Giannoudis et al. also found a difference in outcomes $(p=0.057)$ when comparing Pipkin I and II to III and IV. ${ }^{8}$ A majority of our patients (9/14) with a Pipkin IV fracture underwent ORIF with attention directed toward internal fixation of the acetabular fracture.

$A V N$ is one of the main long-term complications secondary to iatrogenic insult or due to damage during the initial injury. Giannoudis et al. showed an AVN incidence of $11.9 \%$ in their systematic review. ${ }^{8}$ Initially, some surgeons felt the anterior approach should be avoided to protect the blood supply, but a recent meta-analysis and systematic review by Wang et al. showed no difference in rates of AVN between the two approaches. ${ }^{3,17}$ Our series incidence of AVN (9\%) is comparable to the published incidence. One of our patients with AVN had undergone a posterior approach and the other one an anterior approach. Both of these patients required THA eventually. It is important to note our mean follow-up time of 18 months and this time frame may be too short to capture all patients who develop of AVN radiographically. Longer follow-up times are needed to fully quantify AVN rates.

PTA is another well-known complication after injury and occurs secondary to insult to the articular cartilage. The actual incidence is difficult to ascertain given the lack of long term follow up studies. Oransky et al. in a study of femoral head fractures with a mean follow-up of 81 months found that $95.2 \%$ of their patient's 21 femoral head fractures had radiographic criteria of PTA. ${ }^{18}$ In our study, there were 5 patients (23\%) with radiographic arthrosis with two requiring THA; however, this too must be interpreted with respect to our follow-up an average of 18 months. Wang et al. found no difference between anterior or posterior approaches in regard to rates of PTA.17

$\mathrm{HO}$ is a common complication after operative fixation of femoral head fractures with ranges between $6 \%$ and $80 \%{ }^{7,8,16,18-21}$ Surgical approach and time to reduction have been implicated in the process of $\mathrm{HO}$ formation. ${ }^{16,19}$ Wang et al. in a recent systematic review and meta-analysis found that the posterior approach had a statistically significant decrease in the incidence of $\mathrm{HO}$ compared to the anterior approach for Pipkin I and II fractures. ${ }^{17}$ Four of our patients (18\%) developed $\mathrm{HO}$, all with a Brooker grade I. There was no clinical impact of the $\mathrm{HO}$ at the latest clinical follow-up for these four patients

Sciatic nerve damage can occur with an incidence of 3-23\%. ${ }^{7,8,16}$ The peroneal component is the most commonly damaged. Recovery can vary and may take many months. There were two cases of sciatic nerve damage in our study with one patient continuing to exhibit signs of peroneal palsy at latest clinical follow-up. Giannoudis et al. found an infection rate of $3.2 \%$ in 282 operative fractures. One patient (5\%) in our study developed a postoperative infection and this was successfully treated with irrigation and debridement and antibiotics. $^{8}$

There are limitations present in this study. First, our small patient population prevents statistical analysis. Second, our follow-up time can only be categorized as moderate, with an average of 18 months. This is especially important with respect to the complications associated with this injury, namely AVN and PTA, which can take many years to develop. This study should, thus, be interpreted with respect to these limitations.

\section{Conclusion}

Femoral head fractures are rare but potentially devastating injury. Prompt treatment is crucial to preventing long-term outcomes of osteoarthritis and AVN. The principles of treatment include an urgent anatomical reduction, restoration of hip stability, and removal of intra-articular fragments. The present study attempts to further clarify functional outcome results and complication in efforts to add to the growing literature on femoral head fractures associated with hip dislocations. Larger, prospective, randomized studies and the advent of a validated outcomes score are necessary to draw firm conclusions regarding the treatment of femoral head fractures.

\section{Ethical Standards}

This study received IRB approval.

\section{References}

1. Epstein HC. Posterior fracture-dislocations of the hip; longterm follow-up. J Bone Joint Surg Am 1974;56(6):1103-1127. DOI: 10.2106/00004623-197456060-00001.

2. Roeder LF, Jr., DeLee JC. Femoral head fractures associated with posterior hip dislocation. Clin Orthop Relat Res 1980;147:121-130.

3. Epstein HC, Wiss DA, et al. Posterior fracture dislocation of the hip with fractures of the femoral head. Clin Orthop Relat Res 1985;201:9-17.

4. Lang-Stevenson A, Getty CJ. The Pipkin fracture-dislocation of the hip. Injury 1987;18(4):264-269. DOI: 10.1016/0020-1383(87)90011-8.

5. Brumback RJ, Kenzora JE, et al. Fractures of the femoral head. Hip 1987; 181-206.

6. Hougaard K, Thomsen PB. Traumatic posterior fracture-dislocation of the hip with fracture of the femoral head or neck, or both. J Bone Joint Surg Am 1988;70(2):233-239. DOI: 10.2106/00004623-19887002000011.

7. Droll KP, Broekhuyse $\mathrm{H}$, et al. Fracture of the femoral head. J Am Acad Orthop Surg 2007;15(12):716-727. DOI: 10.5435/00124635-20071200000005.

8. Giannoudis PV, Kontakis G, et al. Management, complications and clinical results of femoral head fractures. Injury 2009;40(12): 1245-1251. DOI: 10.1016/j.injury.2009.10.024.

9. Pipkin G. Treatment of grade IV fracture-dislocation of the hip. J Bone Joint Surg Am 1957;39-A(5):1027-1042. DOI: 10.2106/00004623195739050-00004; passim.

10. Brooker AF, Bowerman JW, et al. Ectopic ossification following total hip replacement. incidence and a method of classification. J Bone Joint Surg Am 1973;55(8):1629-1632. DOI: 10.2106/00004623197355080-00006.

11. Thompson VP, Epstein HC. Traumatic dislocation of the hip; a survey of two hundred and four cases covering a period of twenty-one years. J Bone Joint Surg Am 1951;33-A(3):746-778. DOI: 10.2106/00004623195133030-00023; passim.

12. Chen ZW, Lin B, et al. Conservative vs surgical management of Pipkin type I fractures associated with posterior dislocation of the hip: a randomised controlled trial. Int Orthop 2011;35(7):1077-1081. DOI: 10.1007/s00264-010-1087-4.

13. Park KS, Lee KB, et al. Clinical and radiographic outcomes of femoral head fractures: excision vs fixation of fragment in Pipkin type I: what is the optimal choice for femoral head fracture? J Orthop Sci 2015;20(4):702-707. DOI: 10.1007/s00776-015-0732-6. 
14. Holmes WJS, Brian Bay, et al. Biomechanical consequences of excision of displaced Pipkin femoral head fractures. Journal of Orthopaedic Trauma 2000;14(2):149-150. DOI: 10.1097/00005131-20000200000071.

15. Chen ZW, Zhai WL, et al. Operative vs nonoperative management of Pipkin type-II fractures associated with posterior hip dislocation. Orthopedics 2011;34(5):350. DOI: 10.3928/01477447-20110317-09.

16. Marchetti ME, Steinberg GG, et al. Intermediate-term experience of Pipkin fracture-dislocations of the hip. J Orthop Trauma 1996;10(7):455-461. DOI: 10.1097/00005131-199610000-00002.

17. Wang CG, Li YM, et al. Anterior approach vs posterior approach for Pipkin I and II femoral head fractures: a systemic review and metaanalysis. Int J Surg. 2016;27:176-181. DOI: 10.1016/j.ijsu.2016.02.003.

18. Oransky M, Martinelli N, et al. Fractures of the femoral head: a longterm follow-up study. Musculoskelet Surg 2012;96(2):95-99. DOI: 10.1007/s12306-012-0182-7.

19. Swiontkowski MF, Thorpe M, et al. Operative management of displaced femoral head fractures: case-matched comparison of anterior vs posterior approaches for Pipkin I and Pipkin II fractures. J Orthop Trauma 1992;6(4):437-442. DOI: 10.1097/00005131199212000-00008.

20. Henle $P, K$ Kloen $P$, et al. Femoral head injuries: which treatment strategy can be recommended? Injury 2007;38(4):478-488. DOI: 10.1016/ j.injury.2007.01.023.

21. Scolaro JA, Marecek G, et al. Management and radiographic outcomes of femoral head fractures. J Orthop Trauma 2017;18(3):235-241. DOI: 10.1007/s10195-017-0445-z.
22. Asghar FA, Karunakar MA. Femoral head fractures: diagnosis, management, and complications. Orthop Clin North Am 2004;35(4):463-472. DOI: 10.1016/j.ocl.2004.05.004.

23. Butler JE. Pipkin Type-II fractures of the femoral head. J Bone Joint Surg Am 1981;63(8):1292-1296. DOI: 10.2106/00004623-198163080-00011.

24. Chakraborti S, Miller IM. Dislocation of the hip associated with fracture of the femoral head. Injury 1975;7(2):134-142. DOI: 10.1016/00201383(75)90011-X.

25. Gardner MJ, Suk M, et al. Surgical dislocation of the hip for fractures of the femoral head. J Orthop Trauma 2005;19(5):334-342.

26. Marecek GS, Scolaro JA, et al. Femoral Head Fractures. JBJS reviews 2015;3(11), 10.2106/JBJS.RVW.N.00098.

27. Sahin V, Karakas ES, et al. Traumatic dislocation and fracturedislocation of the hip: a long-term follow-up study. J Trauma 2003;54(3):520-509. DOI: 10.1097/01.TA.0000020394.32496.52.

28. Stannard JP, Harris HW, et al. Functional outcome of patients with femoral head fractures associated with hip dislocations. Clin Orthop Relat Res 2000;377:44-56. DOI: 10.1097/00003086-200008000-00008.

29. Stewart MJ, Milford LW. Fracture-dislocation of the hip; an endresult study. J Bone Joint Surg Am 1954;36(A:2):315-342. DOI: 10.2106/00004623-195436020-00010.

30. Tonetti J, Ruatti S, et al. Is femoral head fracture-dislocation management improvable: a retrospective study in 110 cases. Orthop Traumatol Surg Res 2010;96(6):623-631. DOI: 10.1016/j.otsr.2010.03.020.

31. Yoon TR, Rowe SM, et al. Clinical and radiographic outcome of femoral head fractures: 30 patients followed for 3-10 years. Acta Orthop Scand 2001;72(4):348-353. DOI: 10.1080/000164701753541998. 